\title{
Morphologic and immunohistochemical evaluation of splenic hematopoietic proliferations in neoplastic and benign disorders
}

\author{
Dennis P O’Malley ${ }^{1}$, Young S Kim², Sherrie L Perkins ${ }^{3}$, LeeAnn Baldridge ${ }^{1}$, Beth E Juliar ${ }^{4}$ \\ and Attilio Orazi ${ }^{1}$ \\ ${ }^{1}$ Department of Pathology and Laboratory Medicine, Indiana University School of Medicine, Indianapolis, IN, \\ USA; ${ }^{2}$ Department of Pathology, The City of Hope National Medical Center, Duarte, CA, USA; ${ }^{3}$ Department of \\ Pathology, Division of Hematopathology, UUHSC, Salt Lake City, UT, USA and ${ }^{4}$ Division of Biostatistics, \\ Indiana University School of Medicine, Indianapolis, IN, USA
}

\begin{abstract}
Spleen is a common site of extramedullary hematopoiesis. Extramedullary hematopoiesis seen in nonneoplastic conditions can occasionally be extensive and raise concerns for a myeloid neoplasm. We compared the morphologic and immunohistochemical features of splenic hematopoietic proliferations seen in neoplastic myeloid disorders (eg chronic myeloproliferative disorders, myelodysplastic/myeloproliferative disorders and acute myeloid leukemias) to extramedullary hematopoiesis seen in a variety of reactive conditions. In all, $\mathbf{8 0}$ spleen specimens were reviewed. The presence of each marrow-derived lineage, dysplasia and immunohistochemical results were evaluated (CD34, CD117, myeloperoxidase, CD68, p53, TdT, CD42b and hemoglobin). Neoplastic hematopoietic proliferations in chronic myeloproliferative disorders are characterized by trilineage hematopoiesis with significant dysplasia in all cell lineages. Acute myeloid leukemia showed an increase in immature forms, which were highlighted by immunohistochemistry. Reactive extramedullary hematopoiesis showed variability in histologic features. Post-bone marrow transplant and thrombotic thrombocytopenic purpura/hemolytic-uremic syndrome spleens showed extramedullary hematopoiesis with some morphologic features of immaturity, which could simulate chronic myeloproliferative disorder. However, they lacked characteristic immunohistochemical features of neoplastic myeloid disorders such as positivity for CD34 or CD117.

Modern Pathology (2005) 18, 1550-1561. doi:10.1038/modpathol.3800480; published online 19 August 2005
\end{abstract}

Keywords: spleen; extramedullary hematopoiesis; myeloproliferative disorder; neoplastic myeloid disorder

The spleen in humans does not normally function as a site of hematopoiesis. Its role in fetal hematopoiesis is somewhat controversial, and is minimal, at best. ${ }^{1,2}$ However, in normal adult spleen, there is virtually always a very small number of mature hematopoietic elements. The literature supports that these cells are derived from circulating stem cells that take up residence and undergo terminal differentiation, or are circulating maturing elements that are trapped within the spleen, or most likely, a combination of these events. ${ }^{1-3}$

Correspondence: Dr DP O’Malley, MD, Department of Pathology and Laboratory Medicine, Indiana University School of Medicine, 702 Barnhill Dr., Riley 0969, Indianapolis, IN 46202, USA.

E-mail: dpomalle@iupui.edu

Received 29 April 2005; revised 14 July 2005; accepted 15 July 2005; published online 19 August 2005
When benign bone marrow elements are found in an increased number in the spleen, it is termed extramedullary hematopoiesis, which is also referred to as myeloid metaplasia. Extramedullary hematopoiesis is often a result of conditions that disrupt the marrow microenvironment, facilitating the egress of progenitor and precursor cells, with an increase in circulating mature and immature marrow elements. Extramedullary hematopoiesis can be seen in a wide range of splenic conditions varying from benign hematologic conditions, hematopoietic neoplasms, nonhematopoietic tumors and other disorders of the spleen and circulation. ${ }^{4-17}$ The splenic environment is uniquely suited as a site for extramedullary hematopoiesis. It is likely that the stromal microenvironment is similar enough to bone marrow to allow for near-normal development of hematopoietic cell lines. ${ }^{5,7}$ 
However, differentiation of neoplastic hematopoietic proliferations, such as those seen commonly in chronic myeloproliferative disorders, from extramedullary hematopoiesis associated with benign conditions may be difficult in some cases. ${ }^{18}$ In most, the degree of extramedullary hematopoiesis seen in spleens with non-neoplastic conditions or in association with nonhematopoietic neoplasms is inconspicuous enough to not raise concern for possible involvement by a myeloid disease. Occasionally, however, the degree of extramedullary hematopoiesis can be extensive enough to consider a myeloid neoplasm.

The aim of this study was to evaluate the morphologic and immunohistochemical features of neoplastic hematopoietic proliferations in myeloid disorders including chronic myeloproliferative disorders, myelodysplastic/myeloproliferative disorders, myelodysplastic syndromes, systemic mastocytosis and acute myeloid leukemia, and compare those to extramedullary hematopoiesis seen in a variety of reactive conditions or nonhematopoietic tumors of the spleen. Using this information, we then analyzed histologic and immunohistochemical features to evaluate statistical differences between extramedullary hematopoiesis and neoplastic hematopoietic proliferations in the spleen. In addition, we compared histologic and immunohistochemical features of individual entities to see if they could be distinguished from one another based on splenic findings.

\section{Materials and methods}

Materials were obtained from Clarian Health Partners (Indiana University Medical Center, Riley Hospital for Children and Methodist Hospital, Indianapolis, IN, USA), University of Utah Medical Center (Salt Lake City, UT, USA) and City of Hope National Medical Center (Duarte, CA, USA). Files were searched for spleen specimens with either: (1) a diagnosis of a neoplastic myeloid disorder or (2) any spleen with prominent extramedullary hematopoiesis that lacked a diagnosis of a neoplastic, marrow-based disorder. In cases of hematopoietic neoplasms, appropriate blood, bone marrow or other materials were reviewed, when available, to confirm the original diagnosis. Owing to the multi-institutional nature and extreme age of several cases, extensive clinical information was not available for review. Individual tissue blocks with the most prominent or most extensive extramedullary hematopoiesis were selected for further study.

All cases were reviewed and scored by a single author (DPO), with approximately $50 \%$ of cases reviewed and scored by an additional author (AO). Slide reviewers were blinded to the diagnosis at the time of review. H\&E sections were reviewed in each case. The extent of extramedullary hematopoiesis was characterized as: absent $(0+)$, focal $(1+-$ rare clusters of up to five cells), moderate $(2+$ - either several clusters of five to10 cells or any clusters of approximately 50 cells) or extensive $(3+-$ numerous small clusters or areas of diffuse involvement). Cases were graded $0-3+$ for the presence of each marrow-derived lineage (eg myeloid, erythroid, megakaryocytic) based on the method outlined by O'Keane et al. ${ }^{2}$ Each lineage was scored semiquantitatively $(0-3+)$ on the degree of immaturity (erythroid, myeloid) or dysplasia (megakaryocytic) present (Table 1).

Immunohistochemical stains were performed using an automated immunostainer (DakoCytomation, Carpinteria, CA, USA) and a standard streptavidin-biotin-peroxidase complex technique. The following immunohistochemical stains were performed on each case: CD34, CD117, myeloperoxidase, CD68 (PGM-1), p53, TdT, CD42b, hemoglobin and CD8. The manufacturers and staining information are listed in Table 2. Briefly, endogenous peroxidase activity was blocked with $3 \%$ hydrogen peroxide in methanol, and endogenous biotin activity was blocked using avidin and biotin. The peroxidase activity was developed with 3,3-diaminobenzidene, and counterstained with hematoxylin. Control slides and appropriate internal positive controls were reviewed and deemed adequate in all cases.

In the cases of both CD42b and myeloperoxidase, estimation was made of the 'immaturity' or 'dysplasia', respectively, of the positively staining cells and graded on a scale of $0-3+$. In the case of myeloperoxidase, immaturity was defined as positively staining cells with relatively large size, scant cytoplasm and lack of nuclear lobation. 'Dysplasia' of megakaryocytes was defined as abnormally sized megakaryocyte nuclei or abnormalities of nuclear lobation. Any case with blast-sized cells positive for CD42b (eg megakaryoblasts) was scored as $3+$ for dysplasia.

Table 1 Morphologic characteristics of abnormal hematopoietic elements in the spleen

\begin{tabular}{ll}
\hline Lineage & Features \\
\hline Myeloid & $\begin{array}{l}\text { Presence of increased immature/blastic forms } \\
\text { (high N/C ratio, scant cytoplasm, open } \\
\text { chromatin), prominent nucleoli and numerous } \\
\text { hypolobated granulocytes }\end{array}$ \\
Erythroid & $\begin{array}{l}\text { Presence of multinucleated forms, nuclear } \\
\text { fragmentation or budding }\end{array}$ \\
Megakaryocytic & $\begin{array}{l}\text { Clusters of more than three megakaryocytes, } \\
\text { presence of hypolobated forms, hyperlobated } \\
\text { forms, hyperchromatic nuclei, abnormally large } \\
\text { size and abnormally small size (dwarf forms) } \\
\text { The presence of any megakaryoblasts (blast } \\
\text { sized cells with CD42b positivity) was } \\
\text { automatically scored as 3+ for megakaryocytic } \\
\text { dysplasia }\end{array}$
\end{tabular}


Table 2 Immunohistochemical stains

\begin{tabular}{|c|c|c|c|c|}
\hline Antibody & Clone & Manufacturer & Pretreatment & Dilution \\
\hline CD34 & QBEND10 & DakoCytomation & WB/PC & Prediluted \\
\hline CD117 & C-Kit & Oncogene & WB/PC & $1: 50$ \\
\hline CD68 & PGM-1 & DakoCytomation & $\mathrm{E}$ & Prediluted \\
\hline Myeloperoxidase & Polyclonal & DakoCytomation & None & Prediluted \\
\hline P53 & DO.7 & DakoCytomation & WB/PC & Prediluted \\
\hline $\mathrm{TdT}$ & Polyclonal & DakoCytomation & WB/PC & $1: 10$ \\
\hline Hemoglobin & Polyclonal & DakoCytomation & None & $1: 250$ \\
\hline $\mathrm{CD} 42 \mathrm{~b}$ & MM2/174 & Novocastra & WB/PC & $1: 200$ \\
\hline CD8 & CD8/144B & DakoCytomation & WB/PC & Prediluted \\
\hline
\end{tabular}

$\mathrm{WB} / \mathrm{PC}=$ water bath/pressure cooker; $\mathrm{E}=$ enzyme pretreatment.

Novocastra, Newcastle upon Tyne, UK; DakoCytomation, Carpinteria, CA, USA; Oncogene, Boston, MA, USA.

\section{Statistical Analysis}

Data were used to create SAS data sets and analysis was performed in SAS version 8.2 statistical (SAS Institute Inc., Cary, NC, USA) and LogXact version 2.1 software (Cytel Software Corporation, Cambridge, MA, USA). Tests were conducted as twosided with a $P$-value at the 0.05 significance level.

Linear regression was used to predict weight using ordinal predictor for the extent of extramedullary hematopoiesis. Each histologic and immunohistochemical measure was tested for predictive diagnostic value using logistic regression or exact logistic regression (univariate analysis). Models were then tested with all histologic measures and reduced to determine the best set of measures for a diagnosis of benign extramedullary hematopoiesis or a neoplastic myeloid disorder. Subsets of immunohistochemical measures were tested in order to determine a reduced model including only terms with $P$-values $<0.1$. Predictors with $P$-values $<0.05$ were considered significant and $<0.1$ were interpreted as marginally significant. Using best subsets, likelihood ratio test and area under the curve of the receiver operator characteristic curve, a reduced model was determined from all measures. Models with higher area under the curve are interpreted as having higher predictive value.

\section{Results}

A total of 80 spleens were evaluated in the study, 43 with a diagnosis of neoplastic myeloid disorder and 37 with reactive extramedullary hematopoiesis (Table 3). In each case, available clinical data was re-reviewed to confirm the diagnosis and appropriateness for the study.

\section{Demographics}

The age ranges of the patients varied from 2 to 84 years (mean $=51$ years). In the neoplastic group, the range was $4-81$ years (mean $=54$ years), and $2-84$ years (mean $=48$ years) in the reactive group. The weight range was from 56 to $5536 \mathrm{~g}$. The range in the
Table 3 Summary of cases reviewed

\begin{tabular}{lc}
\hline & $\#(\%)$ \\
\hline Neoplastic myeloid disorders & $43(100)$ \\
CMPD & $29(67)$ \\
CIMF & $10(23)$ \\
CML & $11(26)$ \\
PV & $5(12)$ \\
ET & $1(2)$ \\
MDS/MPD & $3(7)$ \\
CMML & $2(5)$ \\
JMML & $1(2)$ \\
MDS & $2(5)$ \\
AML & $9(21)$ \\
Mastocytosis & $2(5)$ \\
Benign & $37(100)$ \\
Autoimmune & $12(32)$ \\
ITP & $4(11)$ \\
AIHA & $3(8)$ \\
TTP/HUS & $6(16)$ \\
Post-BMT & $10(27)$ \\
Other & $2(6)$ \\
Lymphoid neoplasms & \\
\hline
\end{tabular}

$\mathrm{CMPD}=$ chronic myeloproliferative disorder; $\mathrm{CIMF}=$ chronic idiopathic myelofibrosis; $\mathrm{CML}=$ chronic myelogenous leukemia; $\mathrm{PV}=$ polycythemia vera; $\mathrm{ET}=$ essential thrombocythemia; $\mathrm{CMML}=$ chronic myelomonocytic leukemia; JMML = juvenile myelomonocytic leukemia; MDS = myelodysplastic syndrome; AML = acute myeloid leukemia; ITP = immune thrombocytopenic purpura; AIHA = autoimmune hemolytic leukemia; TTP/HUS = thrombotic thrombocytopenic purpura/hemolytic-uremic syndrome; BMT = bone marrow transplant.

neoplastic spleens was 225-5536 g (mean $=1747 \mathrm{~g}$ ), and in the reactive group, the range was 56-2155 g $($ mean $=526 \mathrm{~g})$. Although only seven spleens were from pediatric patients, each of these were at least $3-5 \times$ normal expected weight ranges and would be considered moderate to massive splenomegaly for age. Each of the pediatric spleens reviewed showed findings that were 'typical' for the disorder and did not show any features unique to pediatric patients.

\section{Statistical Evaluation}

\section{Histologic measures}

Not surprisingly, increasing extent of extramedullary hematopoiesis/neoplastic hematopoietic 
proliferation were associated with higher splenic weights $\left(r^{2}=0.292, \beta=0.552\right.$, s.e. $=0.096, P$-value $<0.0001)$. Cases of neoplastic myeloid disorders had greater extent of hematopoietic proliferations compared to reactive extramedullary hematopoiesis (Table 4). Analysis of individual histologic variables by logistic regression analysis suggested that increased levels of the following were associated with neoplastic myeloid disorders: extent of extramedullary hematopoiesis/neoplastic hematopoietic proliferation, number of myeloid elements, myeloid immaturity, number of megakaryocytes and degree of megakaryocytic dysplasia $(P$-values: $<0.0001$, $<0.0001,<0.0001,<0.001$ and $<0.0001$, respectively).

\section{Immunohistochemical measures}

Univariate analysis of immunohistochemical measures revealed that the increases in the following stains were associated with a neoplastic myeloid

Table 4 Extent of EMH in neoplastic myeloid disorders and reactive conditions

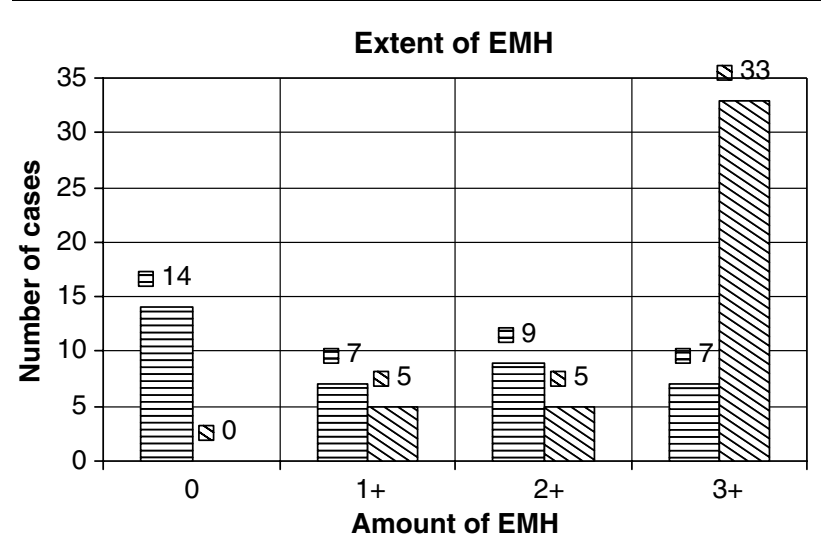

$\boxminus$ Reactive conditions $₫$ Neoplastic myeloid disorders disorder: CD34, CD117, CD42b, number of myeloperoxidase positive and immaturity evaluated by myeloperoxidase ( $P$-values: $0.004,<0.0001,0.005$, $0.001,<0.0001$, respectively). The number of CD68positive cells was of marginal statistical significance in the univariate analysis ( $P$-value: 0.071$)$.

\section{Statistical models}

Table 5 illustrates several models, based on multiple logistic regression, to predict neoplastic myeloid disorders vs reactive extramedullary hematopoiesis. Using histologic features alone, increasing amounts of myeloid elements, megakaryocytic dysplasia and erythroid immaturity favored a diagnosis of a neoplastic myeloid disorder, with an inverse relationship to the number of erythroid elements (area under the curve $=0.928$; $P$-values $<0.0001,0.005$, 0.096 and 0.016 , respectively). In this analysis, increased amounts of erythroid elements favored a diagnosis of reactive extramedullary hematopoiesis.

Using immunohistochemical measures alone, increased numbers of CD34-, CD42b- and myeloperoxidase-positive cells combine to predict neoplastic myeloid disorders (area under the curve $=0.848 ; P$ values: $0.064,0.004$ and 0.024 , respectively). Using a combination of three measures (two histologic, one immunohistochemical), an area under the curve of 0.905 was obtained combining: number of myeloid elements, megakaryocytic dysplasia and number of myeloperoxidase-positive cells. Using four measures, an area under the curve of 0.936 was obtained with: number of myeloid cells, decreased number of erythroid cells, erythroid immaturity and CD42b expression.

\section{Morphologic and Immunohistochemical Profiles of Specific Disease Entities}

The histologic and immunohistochemical results are summarized below and in Tables 6 and 7 .

Table 5 Predictive models of neoplastic myeloid disorders vs reactive EMH

\begin{tabular}{lcll}
\hline Category & $\begin{array}{c}\text { \# of } \\
\text { Predictors }\end{array}$ & Reduced model terms & AUC \\
\hline Histologic parameters & 4 & $\begin{array}{l}\text { Number of myeloid, megakaryocytic dysplasia, erythroid } \\
\text { immaturity and number of erythroid }\end{array}$ & a \\
Immunohistochemical & 3 & CD34, CD42 and number of MPO-positive cells \\
Combined: three predictors & 3 & $\begin{array}{l}\text { Number of myeloid, megakaryocytic dysplasia and number of } \\
\text { MPO-positive cells }\end{array}$ \\
Combined: four predictors & 4 & $\begin{array}{l}\text { Number of myeloid, number of erythroid }{ }^{\text {a }} \text {, erythroid immaturity } \\
\text { and CD42 }\end{array}$
\end{tabular}

AUC $=$ area under the curve (models with higher AUC are interpreted as having higher predictive value); MPO = myeloperoxidase.

${ }^{\mathrm{a}}$ Increased numbers of erythroid elements are less associated with neoplastic myeloid disorders and are more associated with reactive EMH. 
Table 6 Summary of histologic findings in specific disorders

\begin{tabular}{|c|c|c|c|c|}
\hline Disorder & Myeloid & Erythroid & Megakaryocytic & Other \\
\hline CIMF & $\begin{array}{l}++ \\
\text { Immature }\end{array}$ & $+1-$ & $\begin{array}{l}+++ \\
\text { Clusters, dysplastic }\end{array}$ & \\
\hline CML, pattern $1^{\mathrm{a}}$ & $\begin{array}{l}+++ \\
\text { Mature }\end{array}$ & $+1-$ & $+/-$ & $\begin{array}{l}\text { Increased eosinophils and } \\
\text { precursors in some cases }\end{array}$ \\
\hline CML, pattern 2 & +++ & ++ & ++ & \\
\hline P. vera & ++ & +++ & ++ & $\begin{array}{l}\text { Most cases had trilineage } \\
\text { increases suggesting } \\
\text { PPMM }\end{array}$ \\
\hline AML & $\begin{array}{l}+++ \\
\text { Immature }\end{array}$ & $+/-$ & + & $\begin{array}{l}\text { Most cases studied } \\
\text { represented AML } \\
\text { secondary to another } \\
\text { hematologic disorder }\end{array}$ \\
\hline MDS & ++ & $\begin{array}{l}++ \\
\text { Many immature forms }\end{array}$ & $\begin{array}{l}+ \text { to }+++ \\
\text { Dysplastic, hypolobated }\end{array}$ & \\
\hline MDS/MPD & ++ & $+1-$ & - & \\
\hline Mastocytosis & $+1-$ & - & - & \\
\hline Post-BMT & $\begin{array}{l}+++ \\
\text { Predominantly mature } \\
\text { granulocytes and } \\
\text { monocytes }\end{array}$ & + & $+/-$ & \\
\hline TTP/HUS & +++ & ++ & $+1-$ & \\
\hline Erythroid disorders & $\begin{array}{l}+/- \text { to }++ \\
\text { Predominantly mature }\end{array}$ & ++ & - & \\
\hline Splenic lymphoma & $+1-$ & $+1-$ & $+1-$ & \\
\hline
\end{tabular}

$\mathrm{AML}=$ acute myeloid leukemia; $\mathrm{CIMF}=$ chronic idiopathic myelofibrosis; $\mathrm{CML}=$ chronic myelogenous leukemia; $\mathrm{MDS}=$ myelodysplastic syndrome; MDS/MPD = myelodysplastic/myeloproliferative disorders; P. vera = polycythemia vera; post-BMT= post-bone marrow transplant; PPMM = postpolycythemic myeloid metaplasia; TTP/HUS = thrombotic thrombocytopenic purpura/hemolytic-uremic syndrome.

${ }^{\mathrm{a}}$ Two cases of CML were seen in pediatric patients. Each had evidence of $\mathrm{t}(9 ; 22)$ and had histologic findings similar to adult cases.

\section{Myeloproliferative Disorders}

Chronic myeloproliferative disorders represented the single largest group of disorders in the neoplastic myeloid disorder group in our study with 27 cases. These included: 10 cases of chronic idiopathic myelofibrosis (Figure 1), 11 cases of chronic myelogenous leukemia (Figure 2), five cases of polycythemia vera and one case of essential thrombocythemia.

\section{Chronic idiopathic myelofibrosis}

In general, chronic idiopathic myelofibrosis spleens showed extensive, dysplastic trilineage neoplastic hematopoietic proliferations. Prominence of bizarre megakaryocytes, sometimes in clusters of sheets, was common, similar to the descriptions by Thiele et al. ${ }^{19}$ The presence of immature myeloid elements was also extensive in most cases of chronic idiopathic myelofibrosis, suggesting accelerated phase or early transformation to acute myeloid leukemia. CD42b expression was seen in numerous atypical megakaryocytes, and helped to highlight the typically severe nuclear morphologic abnormalities.

\section{Chronic myelogenous leukemia}

Spleens in chronic myelogenous leukemia had two general patterns: the first was predominantly myeloid (eg granulocytic) elements, and the second had bi- or trilineage neoplastic hematopoietic proliferations with increased amounts of megakaryocytic and/or erythroid elements. In 3/11 chronic myelogenous leukemia cases, significant increases in eosinophils and eosinophilic precursors were seen. This finding was not seen in any other type of chronic myeloproliferative disorders, myelodysplastic syndrome, myelodysplastic/myeloproliferative disorders or reactive condition; however, although not seen in our two cases, it would likely be present in cases of mastocytosis. Four cases of chronic myelogenous leukemia had moderate increases in CD68 staining, with faint cytoplasmic staining of cells with a high nuclear/cytoplasmic ratio, suggesting blast cells. These cases also had significant immature granulocytic elements as well. A single case of chronic myelogenous leukemia had increased expression of p53.

\section{Polycythemia vera}

The five polycythemia vera spleens showed varying degrees of neoplastic hematopoietic proliferations, with $3 / 5$ showing extensive involvement. Typically, there were large increases in all three-cell lineages in all cases studied. Based on this finding, these cases probably represented the 'spent' or postpolycythemic myeloid metaplasia phase of polycythemia vera. $^{9}$

\section{Acute myeloid leukemia}

Nine cases of acute myeloid leukemia were studied (23\% of total neoplastic myeloid disorders) (Figure 3). In 7/9 acute myeloid leukemia cases, 
patients had a previous diagnosis of a hematologic disorder, with the spleen removed secondarily. Four of the cases arose in a background of a previous chronic myeloproliferative disorder, two from previous chronic myelomonocytic leukemia and one from a previously diagnosed myelodysplastic syndrome. It is not surprising, given the secondary nature of these cases of acute myeloid leukemia, that

Table 7 Summary of immunohistochemical findings in specific disorders

\begin{tabular}{|c|c|c|c|}
\hline Disorder & Stain & Intensity & Comments \\
\hline \multirow[t]{3}{*}{ CIMF } & $\mathrm{CD} 42 \mathrm{~b}$ & +++ & \\
\hline & MPO & ++ & \\
\hline & CD34/CD117 & $+/-$ & \\
\hline \multirow[t]{2}{*}{ CML } & MPO & +++ & \\
\hline & CD34/CD117 & - to ++ & \\
\hline \multirow{2}{*}{ P. vera } & Hemoglobin & ++ to +++ & \\
\hline & CD34/CD117 & + to ++ & \\
\hline \multirow[t]{5}{*}{ AML } & MPO & ++ to +++ & \\
\hline & CD34 & ++ to +++ & \\
\hline & CD117 & $(++)$ & Present in one case \\
\hline & p53 & $(+)$ & Present in one case \\
\hline & TdT & $(+)$ & Present in one case \\
\hline \multirow[t]{2}{*}{ MDS } & CD34 & ++ & \\
\hline & CD117 & - to ++ & \\
\hline \multirow[t]{4}{*}{ MDS/MPD } & CD68 & ++ & \\
\hline & MPO & ++ & \\
\hline & CD34 & - & \\
\hline & CD117 & - & \\
\hline \multirow[t]{3}{*}{ Mastocytosis } & CD117 & +++ & \\
\hline & MPO & ++ & \\
\hline & CD34 & - & \\
\hline \multirow[t]{4}{*}{ Post-BMT } & MPO & ++ & \\
\hline & CD68 & ++ & \\
\hline & CD34 & - & \\
\hline & CD117 & - & \\
\hline \multirow[t]{4}{*}{ TTP/HUS } & MPO & ++ & \\
\hline & CD68 & ++ & \\
\hline & CD34 & - to $+1-$ & \\
\hline & CD117 & - & \\
\hline \multirow{5}{*}{$\begin{array}{l}\text { Erythroid } \\
\text { disorders }\end{array}$} & MPO & - to ++ & \\
\hline & CD68 & - to + & \\
\hline & Hemoglobin & ++ to +++ & \\
\hline & CD34 & - & \\
\hline & CD117 & - & \\
\hline & MPO & $-/+$ & \\
\hline \multirow[t]{2}{*}{ lymphoma } & CD34 & - & \\
\hline & CD117 & - & \\
\hline
\end{tabular}

$\mathrm{AML}=$ acute myeloid leukemia; $\mathrm{CIMF}=$ chronic idiopathic myelofibrosis; $\quad \mathrm{CML}=$ chronic myelogenous leukemia; $\mathrm{MDS}=$ myelodysplastic syndrome; MDS/MPD = myelodysplastic/myeloproliferative disorders; P. vera $=$ polycythemia vera; post-BMT $=$ post-bone marrow transplant; TTP/HUS = thrombotic thrombocytopenic purpura/hemolytic-uremic syndrome.

Figure 1 Splenic neoplastic hematopoietic proliferation in chronic idiopathic myelofibrosis. (a) H\&E section showing trilineage hematopoietic elements with numerous dysplastic megakaryocytes ( $\times 500$, oil immersion). (b) CD34-positive cells (blasts) and megakaryocytes ( $\times 500$, oil immersion). (c) Only rare positive mast cells are staining for CD117 $(\times 500$, oil immersion). (d) Myeloperoxidase highlights scattered, immature-appearing myeloid cells ( $\times 500$, oil immersion). (e) CD42 highlights clusters of large, dysplastic megakaryocytes ( $\times 500$, oil immersion). there would be considerable overlap in appearance with cases of chronic idiopathic myelofibrosis and other chronic myeloproliferative disorders. All of
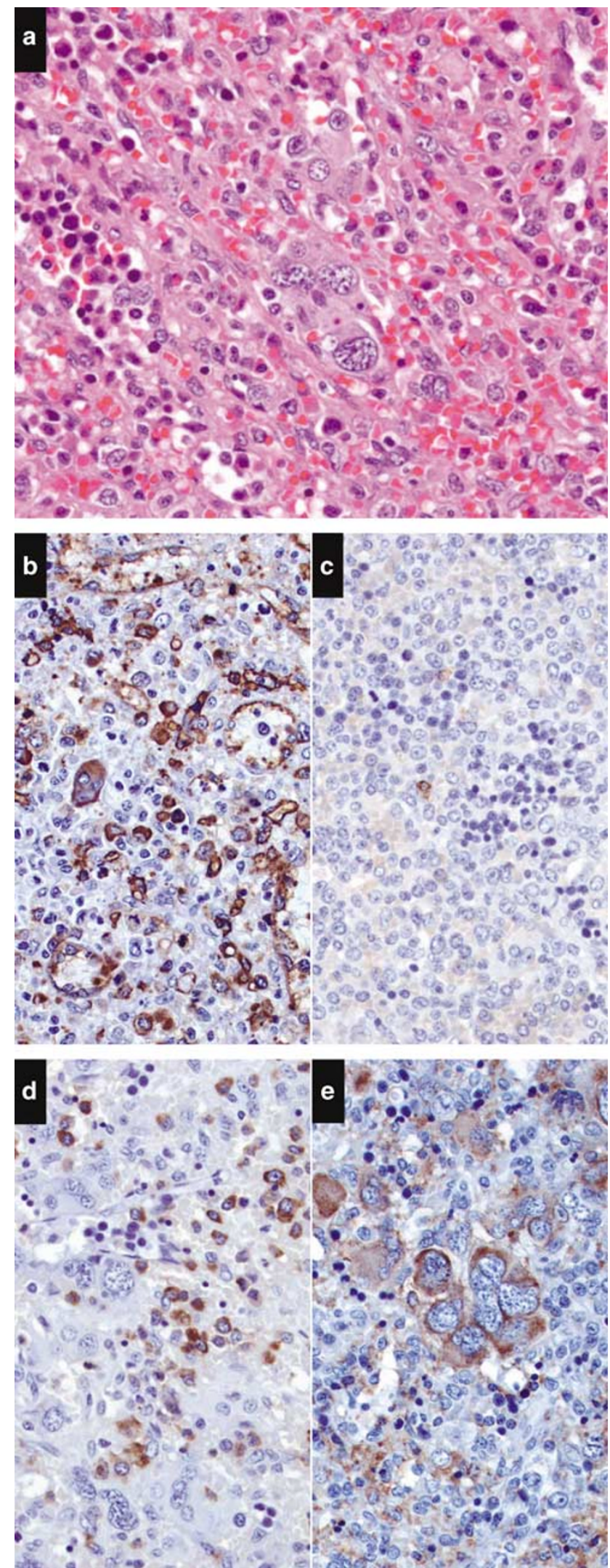
the cases studied had extensive neoplastic hematopoietic proliferations $(3+)$. As expected, all cases studies had extensive myeloid components, all with
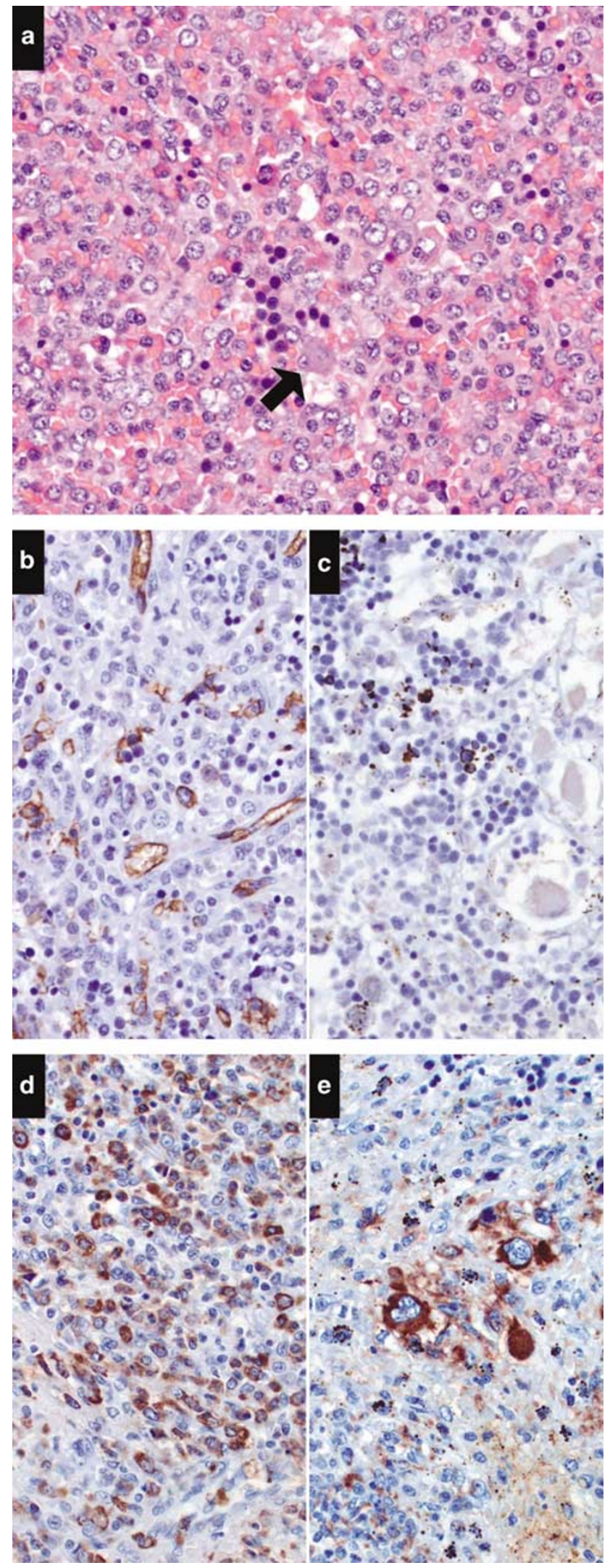

predominantly immature/blastic forms. In addition, several of the cases had significant increases in erythroid and megakaryocytic elements, with associated dysplasia and immaturity noted. Three cases of acute myeloid leukemia had moderate staining for CD68, suggesting monocytic differentiation in at least a subset of neoplastic cells. In one case of myelomonocytic leukemia involving the spleen, numerous blasts were positive for CD68. A single case of acute myeloid leukemia had numerous blasts which expressed CD42b, suggesting megakaryoblastic differentiation. Nuclear p53 expression was seen in a single case of acute myeloid leukemia.

\section{Myelodysplastic syndrome}

Two spleens of patients with myelodysplastic syndrome were analyzed. Both cases showed extensive neoplastic hematopoietic proliferations. In both cases, myeloid components were increased in number with an increase of immature forms. In both cases, the megakaryocytes had abnormal morphology, including small, hypolobated forms, similar to those seen in myelodysplastic syndrome bone marrows. In both cases, the number of CD34 + cells was increased.

\section{Myelodysplastic/myeloproliferative diseases}

Two cases of chronic myelomonocytic leukemia and one case of juvenile myelomonocytic leukemia were evaluated. The two cases of chronic myelomonocytic leukemia had increased numbers of myeloid elements by morphologic exam with increased staining of both CD68 and myeloperoxidase. No immature elements were identified by CD34 or CD117 staining. The single case of juvenile myelomonocytic leukemia analyzed had moderate amounts of predominantly granulocytic hematopoietic proliferation, with no significant expression of CD68, CD34 or CD117. A small amount of erythropoiesis was seen, but no megakaryocytes.

\section{Systemic mastocytosis}

Two cases of mastocytosis were evaluated: one with typical systemic mastocytosis and one with mast cell leukemia. As expected, both cases had significant expression of CD117-positive cells. In both cases, there were numerous myeloperoxidase-positive cells,

Figure 2 Splenic neoplastic hematopoietic proliferation in chronic myelogenous leukemia. (a) H\&E section with numerous granulocytes, rare erythroid elements and occasional small megakaryocytes (arrow) ( $\times 500$, oil immersion). (b) Rare, scattered CD34-positive immature cells and positive vascular elements ( $\times 500$, oil immersion). (c) No increased numbers of CD117positive cells were seen in chronic myelogenous leukemia cases in this study $(\times 500$, oil immersion). (d) Myeloperoxidase staining highlights numerous granulocytic precursors $(\times 500$, oil immersion). (e) CD42 staining in a cluster of megakaryocytes $(\times 500$, oil immersion). 
but these represented mature granulocytic elements. Neither benign nor malignant mast cells express myeloperoxidase. ${ }^{20}$
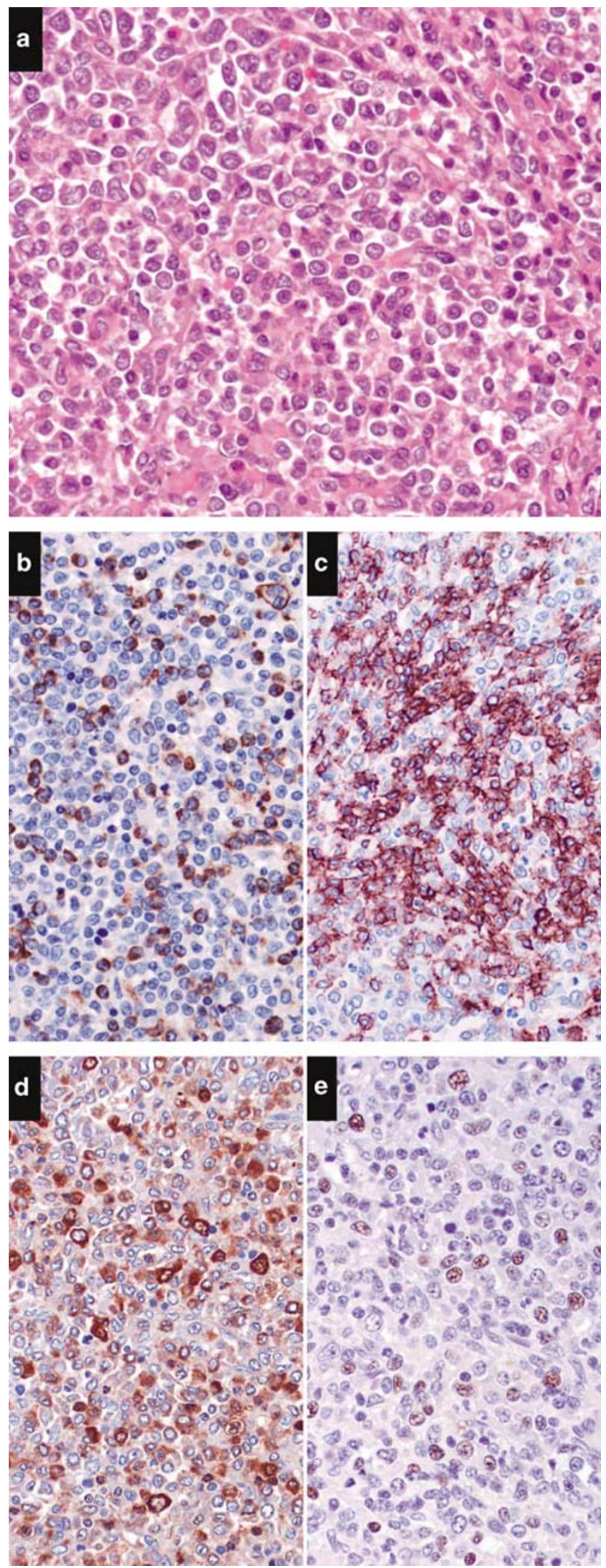

\section{Reactive Extramedullary Hematopoiesis}

\section{Post-bone marrow transplant}

Six splenectomies associated with post-bone marrow transplant had moderate to extensive extramedullary hematopoiesis (Figure 4). Although predominantly composed of myeloid elements, with myeloperoxidase and CD68 positivity, erythroid elements were often prominent, and megakaryocytes were present to a lesser degree. A single case expressed a mild increase in CD34 expression. Significant increases in erythroid precursors were highlighted by the hemoglobin immunohistochemical stain.

\section{Thrombotic thrombocytopenic purpura/ hemolytic-uremic syndrome}

Three cases of thrombotic thrombocytopenic purpura/hemolytic-uremic syndrome had surprisingly high levels of extramedullary hematopoiesis. The cases had either moderate to extensive amounts, which consisted significant numbers of erythroid and myeloid elements, with increased megakaryocytes in two cases (Figure 5). A single case expressed a mild increase in CD34 expression. Hemoglobin staining highlighted numerous erythroid precursors.

\section{Erythroid disorders associated with hemolysis}

Seven cases of primarily erythroid disorders were studied including four cases of autoimmune hemolytic anemia, two hereditary spherocytosis and one thalassemia. Only three of the seven cases showed significant extramedullary hematopoiesis, not surprisingly with extensive amounts of erythroid elements, both by morphologic examination and by hemoglobin staining. In addition, rare to large numbers of myeloid elements were also seen, both by histology and by myeloperoxidase staining.

\section{Splenic lymphoma}

Two cases of splenic B-cell lymphoma (one splenic marginal zone lymphoma, one low-grade B-cell lymphoma, not otherwise classified) had focal $(1+)$ extramedullary hematopoiesis. This consisted of small amounts of myeloid, erythroid and megakaryocytic elements, scattered throughout the red pulp.

Figure 3 Splenic neoplastic hematopoietic proliferation in acute myeloid leukemia. (a) H\&E illustrating infiltration of the splenic red pulp by a relatively homogeneous population of blastic hematopoietic cells $(\times 500$, oil immersion). (b) CD34 staining in acute myeloid leukemia in the spleen $(\times 500$, oil immersion $)$. (c) Rare cases of acute myeloid leukemia strongly expressed CD117, as in this case $(\times 500$, oil immersion). (d) Myeloperoxidase staining in a case of acute myeloid leukemia $(\times 500$, oil immersion). (e) p53 staining in a case of acute myeloid leukemia $(\times 500$, oil immersion). 

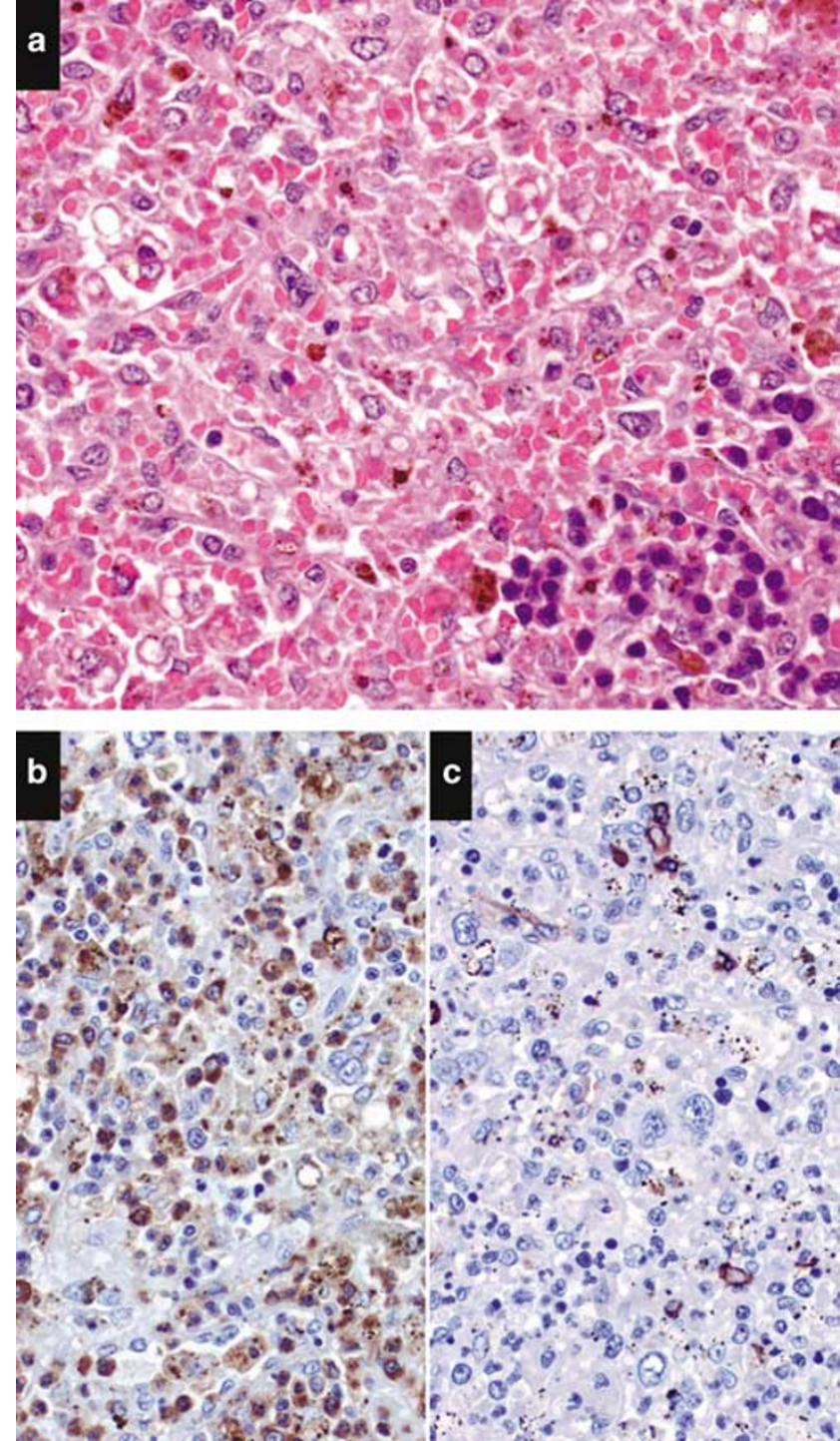

Figure 4 Splenic extramedullary hematopoiesis in post-bone marrow transplant. (a) H\&E section illustrating numerous monocytes, macrophages and erythroid precursors $(\times 500$, oil immersion). (b) Myeloperoxidase staining highlights the numerous myeloid elements in postbone marrow transplant marrows ( $\times$ 500, oil immersion). (c) In contrast to many neoplastic myeloid disorders, no increase in CD34-positive cells is seen in post-bone marrow transplant $(\times 500$, oil immersion $)$.

\section{Other reactive extramedullary hematopoiesis}

The other cases included splenectomy specimens for a wide variety of conditions including immune thrombocytopenic purpura $(n=12)$, trauma $(n=2)$, fibrocongestive splenomegaly $(n=1)$, severe combined immunodeficiency $(n=1)$ and two cases with no specific diagnosis but with extramedullary hematopoiesis. In immune thrombocytopenic purpura, all but one case showed the extent of extramedullary hematopoiesis at extremely low levels $(0+)$, with only rare, scattered erythroid elements or mature megakaryocytes. The other reactive cases varied widely in the extent and appearance of extramedullary hematopoiesis. Only
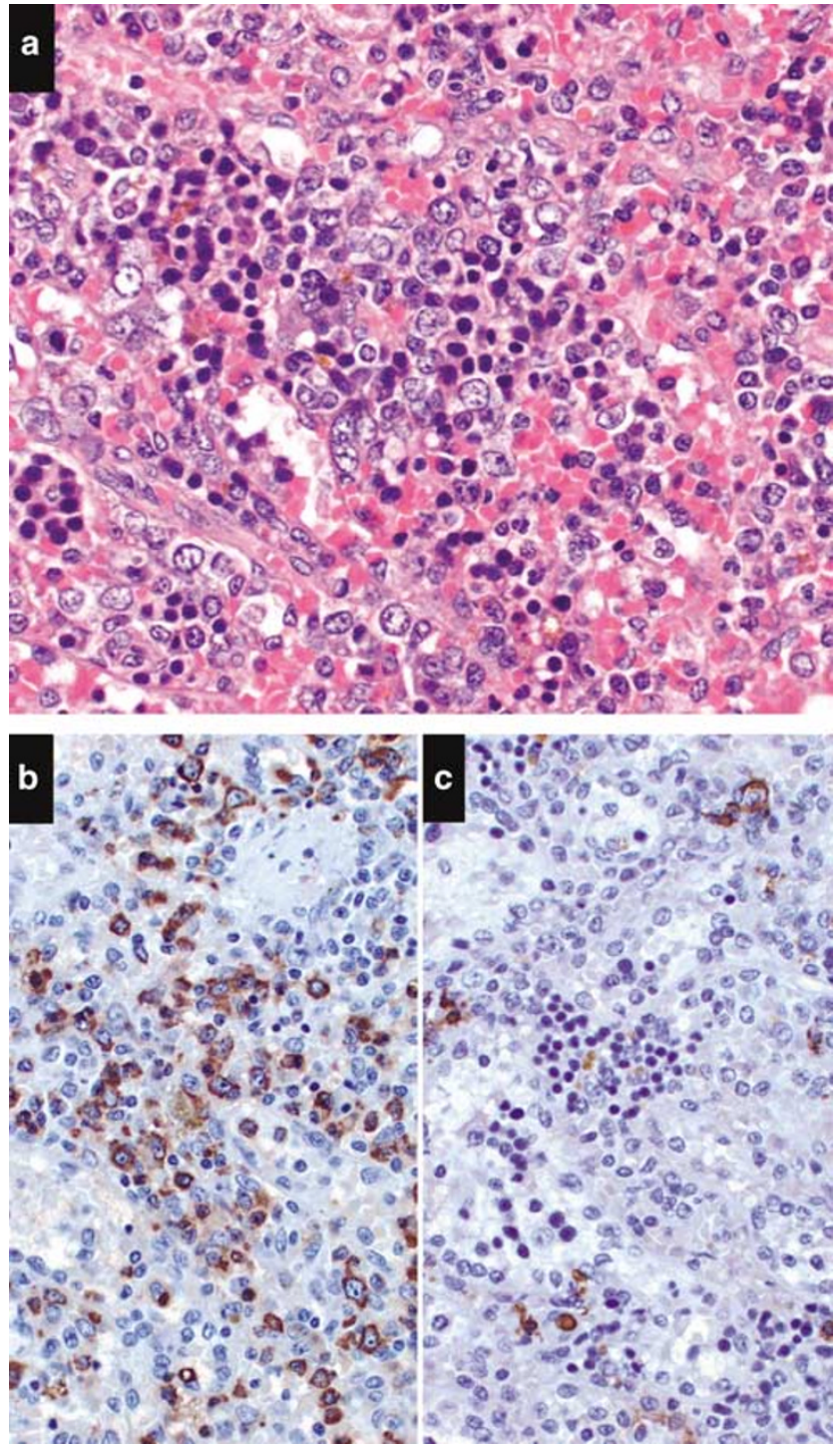

Figure 5 Splenic extramedullary hematopoiesis in hemolyticuremic syndrome. (a) H\&E section illustrates immature-appearing myeloid and erythroid elements, similar to that seen in thrombotic thrombocytopenic purpura ( $\times 500$, oil immersion). (b) Myeloperoxidase confirms the myeloid nature of the immature cells ( $\times 500$, oil immersion). (c) No CD34 expression is seen in the infiltrate in hemolytic-uremic syndrome or thrombotic thrombocytopenic purpura $(\times 500$, oil immersion $)$.

rare granulocytic and monocytic/macrophage elements were detected by immunohistochemical with myeloperoxidase and CD68. With the exception of one case of immune thrombocytopenic purpura with aberrant staining for CD34 in some cells, no significant CD34 or CD117 was seen in these cases of reactive extramedullary hematopoiesis.

\section{Conclusions}

A variety of factors complicate evaluation of splenic pathology. Quality of fixation and processing, 
relative rarity of splenectomy specimens and a complex histology combining several functional capacities in a single organ all add to the difficulties encountered by the pathologist. Although a relatively common pathologic finding, splenic extramedullary hematopoiesis can occasionally present diagnostic difficulties. These difficulties were highlighted in this study by certain entities, which lack the clearcut clinical or histologic features of either obvious neoplastic hematopoietic proliferation or reactive extramedullary hematopoiesis.

In this study, we were able to demonstrate that by using both morphology and immunohistochemistry in combination, it is possible to distinguish between reactive extramedullary hematopoiesis and that seen in neoplastic myeloid disorders. The statistical data support our initial impressions that certain histologic features are seen more commonly in neoplastic myeloid disorders than in reactive extramedullary hematopoiesis. Those features seen in the spleens of neoplastic myeloid disorders include increasing amounts of myeloid elements and immaturity of the hematopoietic elements present. Perhaps not surprisingly, an increase in the number of erythroid elements, especially as an isolated finding, seems to support the presence of reactive extramedullary hematopoiesis, at least in most cases. By immunohistochemistry, an increased number of immature hematopoietic elements (by CD34 and CD117), of myeloperoxidase-positive myeloid cells, and CD42b-positive megakaryocytes were all significantly associated with neoplastic myeloid proliferations.

In many of our cases of chronic myeloproliferative disorder-associated acute myeloid leukemia, the histologic and immunohistochemical findings were indistinguishable from cases of chronic idiopathic myelofibrosis. In cases with increased immature myeloid elements and dysplastic megakaryocytes, splenic pathology alone may not adequately distinguish transformation to acute myeloid leukemia from chronic myeloproliferative disorders. In these circumstances, clinical information and concurrent blood and bone marrow findings play an important role in this distinction of acute transformation. It is known that spleen is a common site of transformation of chronic idiopathic myelofibrosis and other chronic myeloproliferative disorders to acute myeloid leukemia. It is quite likely that there is sampling bias in this study, since chronic idiopathic myelofibrosis spleens were removed usually symptomatic, possibly due to an accelerated disease course. In a study by Mesa et $a l^{21}$ of hematopoietic cells in chronic idiopathic myelofibrosis, those with predominantly immature precursors (eg blasts) had worse overall survival.

Our cases of chronic myelogenous leukemia showed a broad range of morphologic features. Some of the cases of chronic myelogenous leukemia had more immature elements by morphology and immunohistochemistry compared to others. These cases may also have represented accelerated phases or blastic transformations of chronic myelogenous leukemia. However, as in chronic idiopathic myelofibrosis cases, this may be due to a similar selection bias, namely that splenectomy is performed in symptomatic patients and may be a result of worsening symptoms or cytopenias, and may herald a transformation to acute myeloid leukemia.

Wolf et $a l^{9}$ noted that the erythrocytotic phase of polycythemia vera is associated with only minimal hematopoietic proliferations, consisting of mostly erythroid elements and rare megakaryocytes. The increase in size of the spleen associated with this disorder was considered by these authors as predominantly due to congestion. In the current study, almost all polycythemia vera spleens showed increases in myeloid, erythroid and megakaryocytic elements. The cases in our study represented postpolycythemic myeloid metaplasia, an advanced or 'spent' stage of polycythemia vera. In postpolycythemic myeloid metaplasia, like chronic idiopathic myelofibrosis, the splenic enlargement is due in part to neoplastic hematopoietic proliferation as well as proliferation of stromal elements.

Kraus et $a l^{16}$ described splenic changes in myelodysplastic syndrome and chronic myelomonocytic leukemia. All cases studied had expansion of the red pulp and trilineage hematopoiesis. Four patterns of splenic pathology were identified: erythroid extramedullary hematopoiesis with erythrophagocytosis, red pulp congestion with plasmacytosis, extramedullary hematopoiesis with red pulp congestion and dyserythropoiesis, and a chronic myelomonocytic leukemia pattern. Kraus ${ }^{22}$ concluded that the cellular composition of myelodysplastic syndrome cannot be reliably distinguished from chronic myeloproliferative disorders based on histologic section. We found this to be generally true of all the neoplastic myeloid disorders included in the study, although there were some notable differences including marked eosinophilia in rare cases of chronic myelogenous leukemia, fibrosis and peritrabecular distribution in mastocytosis, increased numbers of immature cells in acute myeloid leukemia and the numerous atypical megakaryocytes seen in chronic idiopathic myelofibrosis.

Among the cases of reactive extramedullary hematopoiesis, two subgroups were notable in this study, due to the presence of large amounts of extramedullary hematopoiesis: postbone marrow transplant and thrombotic thrombocytopenic purpura/hemolytic-uremic syndrome. Prominent extramedullary hematopoiesis has been described on both conditions previously, ${ }^{5,10,12,13}$ but none of these studies described the cell composition in detail. In postbone marrow transplant patients, the spleen serves as a site of hematopoietic reconstitution. Although morphology suggests immaturity, the lack 
of markers of immature myeloid proliferations (CD34, CD117) is reassuring in the identification of benign disorders. In these cases, clinical histories, and an awareness of the extent of extramedullary hematopoiesis that can be seen, are the most critical features to prevent a possible misdiagnosis. Although described in previous studies, satisfactory explanations as to the extent of splenic extramedullary hematopoiesis in thrombotic thrombocytopenic purpura/hemolytic-uremic syndrome are lacking. Consumption of platelets and red blood cells by the disease process suggests that the precursor elements expand in response to this. However, splenomegaly, increases in myeloid elements, and the large increases in extent of extramedullary hematopoiesis are not seen in other comparable conditions, and may represent a relatively unique, albeit rare, feature of thrombotic thrombocytopenic purpura/hemolytic-uremic syndrome.

Morphologic evaluation of hematopoietic proliferations in spleen may lead to underestimation or misidentification of marrow elements within the complex histologic makeup of the spleen. Likewise, immunohistochemical evaluation may lead to overestimation or misidentification of myeloid elements such as staining patterns with high background positivity (ie megakaryocytic stains/platelets, myeloperoxidase/mature granulocytes and monocytes). Our statistical evaluation shows that several individual histologic and immunohistochemical features are significant predictors in distinguishing neoplastic myeloid disorders from reactive extramedullary hematopoiesis in the spleen. We have found that a combination of histologic and immunohistochemical evaluation provides the best method for evaluating splenic hematopoietic proliferations. Our results suggest that, taken in concert, it is possible to evaluate the distribution and amount of extramedullary hematopoiesis, identify the lineage and make estimates of cellular maturity. We recommend that if immunohistochemical stains are used, a panel including CD34, CD117 and myeloperoxidase, possibly with the inclusion of a megakaryocytespecific marker (eg CD42b), may clarify difficult diagnoses.

It is our experience that splenectomy specimens, even with a known diagnosis of an underlying hematologic disorder, often provide diagnostic challenges to the practicing pathologist. When splenectomies are performed, it is usually for the purposes of relief of symptoms. However, several factors may complicate this clinical decision including the possible presence of: infarction, infection and blastic transformations/acceleration of hematologic disease.

Although there remain difficult cases with features that overlap benign extramedullary hematopoiesis and neoplastic hematopoietic proliferations, careful application of histologic examination and select immunohistochemical stains will often distinguish these cases.

\section{Acknowledgement}

We thank Richard S Neiman, MD for his review and helpful suggestions on this manuscript.

\section{References}

1 Freedman MH, Saunders EF. Hematopoiesis in the human spleen. Am J Hematol 1981;11:271-275.

2 O'Keane JC, Wolf BC, Neiman RS. The pathogenesis of splenic extramedullary hematopoiesis in metastatic carcinoma. Cancer 1989;63:1539-1543.

3 Wilkins BS, Green A, Wild AE, et al. Extramedullary haemopoiesis in fetal and adult human spleen: a quantitative immunohistological study. Histopathology 1994;24:241-247.

4 Snover DC, Frizzera G, Spector BD, et al. WiskottAldrich syndrome: histopathologic findings in the lymph nodes and spleens of 15 patients. Hum Pathol 1981;12:821-831.

5 Arnold R, Calvo W, Heymer B, et al. Extramedullary hematopoiesis after bone marrow transplantation. Scan J Haematol 1985;34:9-12.

6 Hassan NM, Neiman RS. The pathology of the spleen in steroid-treated immune thrombocytopenic purpura. Am J Clin Pathol 1985;84:433-438.

7 Douay L, Laporte JP, Lefrancois G, et al. Blood and spleen haematopoiesis in patients with myelofibrosis. Leukemia Res 1987;11:725-730.

8 Palitzsch KD, Falk S, Muller H, et al. Splenic haematopoiesis in patients with cirrhosis of the liver. Virchows Arch 1987;411:179-183.

9 Wolf BC, Banks PM, Mann RB, et al. Splenic hematopoiesis in polycythemia vera. A morphologic and immunohistologic study. Am J Clin Pathol 1988;89:69-75.

10 Dilly SA, Sloane JP. Cellular composition of the spleen after human allogeneic bone marrow transplantation. J Pathol 1988;155:151-160.

11 Mathew A, Raviglione MC, Niranjan U, et al. Splenectomy in patients with AIDS. Am J Hematol 1989;32: 184-189.

12 Horny H-P, Ruck M, Kaiserling E. Immunohistology of the human spleen after bone marrow transplantation for leukemia with special reference to the early posttransplantation period. Pathol Res Pract 1990;186: 775-783.

13 Saracco SM, Farhi DC. Splenic pathology in thrombotic thrombocytopenic purpura. Am J Surg Pathol 1990; 14:223-229.

14 Chang CS, Li CY, Cha SS. Chronic idiopathic thrombocytopenic purpura. Splenic pathologic features and their clinical correlation. Arch Pathol Lab Med 1993; 117:981-985.

15 Farhi DC, Ashfaq R. Splenic pathology after traumatic injury. Am J Clin Pathol 1996;105:474-478.

16 Kraus MD, Bartlett NL, Fleming MD, et al. Splenic pathology in myelodysplasia. A report of 13 cases with clinical correlation. Am J Surg Pathol 1998;22: 1255-1266.

17 Steensma DP, Tefferi A, Li C. Splenic histopathological patterns in chronic myelomonocytic leukemia with clinical correlations: reinforcement of the heterogeneity of the syndrome. Leukemia Res 2003;27:775-782.

18 Neiman RS, Orazi A. Myeloproliferative disorders. In: Disorders of the Spleen, 2nd edn. WB Saunders: Philadelphia, 1999, pp 220-236. 
19 Thiele J, Klein H, Falk S, et al. Splenic megakaryocytopoiesis in primary (idiopathic) osteomyelofibrosis. An immunohistological and morphometric study with comparison of corresponding bone marrow features. Acta Haematol 1992;87: 176-180.

20 Horny HP, Valent P. Diagnosis of mastocytosis: general histopathological aspects, morphological criteria, and immunohistochemical findings. Leukemia Res 2001; 25:543-551.

21 Mesa RA, Li CY, Schroeder G, et al. Clinical correlates of splenic histopathology and splenic karyotype in myelofibrosis with myeloid metaplasia. Blood 2001; 97:3665-3667.

22 Kraus MD. Splenic histology and histopathology: an update. Semin Diagn Pathol 2003;20:84-93. 